\title{
On the Fading Number of Multiple-Input Single-Output Fading Channels with Memory
}

\author{
Stefan M. Moser \\ Department of Communication Engineering \\ National Chiao Tung University (NCTU) \\ Hsinchu, Taiwan \\ Email: stefan.moser@ieee.org
}

\begin{abstract}
We derive new upper and lower bounds on the fading number of multiple-input single-output (MISO) fading channels of general (not necessarily Gaussian) regular law with spatial and temporal memory. The fading number is the second term, after the double-logarithmic term, of the high signal-tonoise ratio (SNR) expansion of channel capacity.

In case of an isotropically distributed fading vector it is proven that the upper and lower bound coincide, i.e., the general MISO fading number with memory is known precisely.

The upper and lower bounds show that a type of beam-forming is asymptotically optimal.
\end{abstract}

KEYWORDS: Beam-forming, channel capacity, fading, fading number, high SNR, memory, MISO, multiple-antenna.

\section{INTRODUCTION}

It has been recently shown in [1], [2] that, whenever the matrix-valued fading process is of finite differential entropy rate, the capacity of multiple-input multiple-output (MIMO) fading channels typically grows only double-logarithmically in the signal-to-noise ratio (SNR). To quantify the rates at which this poor power efficiency begins, [1], [2] introduced the fading number as the second term in the high-SNR asymptotic expansion of channel capacity. Explicit expressions for the fading number were then given for a number of fading models. For channels with memory, the fading number of single-input single-output (SISO) fading channels was derived in [1], [2] and the single-input multiple-output (SIMO) case was derived in [3], [4], [2].

The fading number of the multiple-input single-output (MISO) fading channel has been derived in general only for the memoryless case [1], [2]:

$$
\chi\left(\mathbf{H}^{\top}\right)=\sup _{\|\hat{\mathbf{x}}\|=1}\left\{\log \pi+\mathrm{E}\left[\log \left|\mathbf{H}^{\top} \hat{\mathbf{x}}\right|^{2}\right]-h\left(\mathbf{H}^{\top} \hat{\mathbf{x}}\right)\right\} .
$$

This fading number is achievable by inputs that can be expressed as the product of a constant unit vector in $\mathbb{C}^{n_{\mathrm{T}}}$ and a circularly symmetric, scalar, complex random variable of the same law that achieves the memoryless SISO fading number [1]. Hence, the asymptotic capacity of a MISO fading channel is achieved by beam-forming where the beam-direction is chosen not to maximize the SNR, but the fading number.

In [5] and [6] Koch \& Lapidoth investigate the fading number of MISO fading channels with memory where the fading is Gaussian. For the case of a mean-d Gaussian vector process with memory where $\left\{\mathbf{H}_{k}-\mathbf{d}\right\}$ is spatially independent and identically distributed (IID) and where each component is a zero-mean unit-variance circularly symmetric complex Gaussian process, the fading number is shown to be ${ }^{1}$

$$
\begin{aligned}
\chi_{\text {Gauss, spat. IID }}\left(\left\{\mathbf{H}_{k}^{\top}\right\}\right)= & -1+\log \|\mathbf{d}\|^{2}-\operatorname{Ei}\left(-\|\mathbf{d}\|^{2}\right) \\
& +\log \frac{1}{\epsilon^{2}},
\end{aligned}
$$

where $\epsilon^{2}$ denotes the prediction error when predicting one of the components of the fading vector based on the observation of its past.

Furthermore, Koch \& Lapidoth derive an upper bound to the fading number for the general Gaussian case, i.e., $\left\{\mathbf{H}_{k}-\mathbf{d}\right\}$ is a zero-mean circularly symmetric stationary ergodic complex Gaussian process with matrix-valued spectral distribution function $F(\cdot)$ and with covariance matrix K. Assuming that the prediction error covariance matrix $\Sigma$ is non-singular (regularity assumption) they show that

$$
\chi_{\text {Gauss }}\left(\left\{\mathbf{H}_{k}^{\top}\right\}\right) \leq-1+\log d_{*}^{2}-\operatorname{Ei}\left(-d_{*}^{2}\right)+\log \frac{\|\mathrm{K}\|}{\lambda_{\min }},
$$

where

$$
d_{*}=\max _{\|\hat{\mathbf{x}}\|=1} \frac{\left|\mathrm{E}\left[\mathbf{H}_{k}^{\top}\right] \hat{\mathbf{x}}\right|}{\sqrt{\operatorname{Var}\left(\mathbf{H}_{k}^{\top} \hat{\mathbf{x}}\right)}}
$$

$\lambda_{\min }$ denotes the smallest eigenvalue of $\Sigma$; and where $\|\cdot\|$ denotes the Euclidean operator norm of matrices, i.e., the largest singular value.

In this paper we extend these results to general (not necessarily Gaussian) fading channels.

The remaining of this paper is structured as follows: after defining the channel model in detail in the following section, we will present the main results, i.e., a new upper and lower bound on the MISO fading number, in Section III

We then specialize these results to the case of isotropically distributed fading processes in Section IV and to Gaussian fading in Section V] For isotropically distributed fading we will show that the upper and lower bound coincide. In the Gaussian case we shall derive the above mentioned results of Koch \& Lapidoth as special cases of our bounds.

We conclude in Section VI

\footnotetext{
${ }^{1}$ Note that all results in this paper are in nats.
} 


\section{The Channel Model}

We consider a MISO fading channel whose time- $k$ output $Y_{k} \in \mathbb{C}$ is given by

$$
Y_{k}=\mathbf{H}_{k}^{\top} \mathbf{x}_{k}+Z_{k}
$$

where $\mathbf{x}_{k} \in \mathbb{C}^{n_{\mathrm{T}}}$ denotes the time- $k$ channel input vector; where the random vector $\mathbf{H}_{k}$ denotes the time- $k$ fading vector; where $\mathbf{H}_{k}^{\top}$ denotes the transpose of the vector $\mathbf{H}_{k}$; and where $Z_{k}$ denotes additive noise. Here $\mathbb{C}$ denotes the complex field, $\mathbb{C}^{n_{\mathrm{T}}}$ denotes the $n_{\mathrm{T}}$-dimensional complex Euclidean space, and $n_{\mathrm{T}}$ is the number of transmit antennas. We assume that the additive noise is an IID zero-mean white Gaussian process of variance $\sigma^{2}>0$.

As for the multi-variate fading process $\left\{\mathbf{H}_{k}\right\}$, we shall only assume that it is stationary, ergodic, of finite second moment

$$
\mathrm{E}\left[\left\|\mathbf{H}_{k}\right\|^{2}\right]<\infty
$$

and of finite differential entropy rate

$$
h\left(\left\{\mathbf{H}_{k}\right\}\right)>-\infty .
$$

Finally, we assume that the fading process $\left\{\mathbf{H}_{k}\right\}$ and the additive noise process $\left\{Z_{k}\right\}$ are independent and of a joint law that does not depend on the channel input $\left\{\mathbf{x}_{k}\right\}$.

As for the input, we consider two different constraints: a peak-power constraint and an average-power constraint. We use $\mathcal{E}$ to denote the maximal allowed instantaneous power in the former case, and to denote the allowed average power in the latter case. For both cases we set

$$
\mathrm{SNR} \triangleq \frac{\mathcal{E}}{\sigma^{2}}
$$

The capacity $\mathrm{C}(\mathrm{SNR})$ of the channel (5) is given by

$$
\mathrm{C}(\mathrm{SNR})=\lim _{n \rightarrow \infty} \frac{1}{n} \sup I\left(\mathbf{X}_{1}^{n} ; Y_{1}^{n}\right)
$$

where we use $\mathbf{X}_{j}^{k}$ to denote $\mathbf{X}_{j}, \ldots, \mathbf{X}_{k}$ and where the supremum is over the set of all probability distributions on $\mathbf{X}_{1}^{n}$ satisfying the constraints, i.e.,

$$
\left\|\mathbf{X}_{k}\right\|^{2} \leq \mathcal{E}, \quad \text { almost surely, } \quad k=1,2, \ldots, n
$$

for a peak constraint, or

$$
\frac{1}{n} \sum_{k=1}^{n} \mathrm{E}\left[\left\|\mathbf{X}_{k}\right\|^{2}\right] \leq \mathcal{E}
$$

for an average constraint.

Specializing [1, Theorem 4.2] or [2, Theorem 6.10], respectively, to MISO fading, we have

$$
\varlimsup_{\operatorname{SNR} \uparrow \infty}\{\mathrm{C}(\mathrm{SNR})-\log \log \mathrm{SNR}\}<\infty .
$$

The fading number $\chi$ is now defined as in [1, Definition 4.6] and in [2, Definition 6.13] by

$$
\chi\left(\left\{\mathbf{H}_{k}^{\top}\right\}\right) \triangleq \varlimsup_{\text {SNR } \uparrow \infty}\{\mathrm{C}(\mathrm{SNR})-\log \log \mathrm{SNR}\} .
$$

Prima facie the fading number depends on whether a peakpower constraint (10) or an average-power constraint (11) is imposed on the input. Since a peak-power constraint is more stringent than an average-power constraint, we will derive the upper bound using the average-power constraint and the lower bound using the peak-power constraint. In case of an isotropically distributed fading process we shall see that both constraints lead to identical fading numbers.

\section{Main Results}

We first state a new upper bound to the fading number of a MISO fading channel:

Theorem 1: Consider a MISO fading channel with memory (5) where the stationary and ergodic fading process $\left\{\mathbf{H}_{k}\right\}$ takes value in $\mathbb{C}^{n_{\mathrm{T}}}$ and satisfies $h\left(\left\{\mathbf{H}_{k}\right\}\right)>-\infty$ and $\mathrm{E}\left[\left\|\mathbf{H}_{k}\right\|^{2}\right]<\infty$. Then, irrespective of whether a peak-power constraint (10) or an average-power constraint (11) is imposed on the input, the fading number $\chi\left(\left\{\mathbf{H}_{k}^{\top}\right\}\right)$ is upper-bounded by

$$
\begin{aligned}
\chi\left(\left\{\mathbf{H}_{k}^{\top}\right\}\right) \leq \sup _{\hat{\mathbf{x}}_{-\infty}^{0}}\left\{\log \pi+\mathrm{E}\left[\log \left|\mathbf{H}_{0}^{\top} \hat{\mathbf{x}}_{0}\right|^{2}\right]\right. \\
\left.-h\left(\mathbf{H}_{0}^{\top} \hat{\mathbf{x}}_{0} \mid\left\{\mathbf{H}_{\ell}^{\top} \hat{\mathbf{x}}_{\ell}\right\}_{\ell=-\infty}^{-1}\right)\right\}
\end{aligned}
$$

where $\hat{\mathbf{x}}_{\ell} \triangleq \frac{\mathbf{x}_{\ell}}{\left\|\mathbf{x}_{\ell}\right\|}$ denotes a vector of unit length.

Proof: The proof is in part pretty technical. We therefore give only an outline and omit the details.

Similar to the derivation of the SIMO fading number with memory, the proof starts with a lemma that limits the possible joint input distributions on $\mathbf{X}_{1}, \ldots, \mathbf{X}_{n}$ to such under which each random vector $\mathbf{X}_{\ell}$ has the same law with an average power equal to the constraint $\mathcal{E}$. Unfortunately, the proof is complicated by the fact that this lemma does not guarantee equal marginals for the time epochs $k$ on the border of a block. However, these edge effects wash out once we let the blocklength $n$ tend to infinity.

The proof then proceeds as follows: the mutual information between joint input and joint output is split up into a term describing the memoryless case and a term that takes care of the memory:

$$
\begin{aligned}
\lim _{n \rightarrow \infty} & \frac{1}{n} I\left(\mathbf{X}_{1}^{n} ; Y_{1}^{n}\right) \\
\leq \lim _{n \rightarrow \infty} \frac{1}{n} \sum_{k=1}^{n} & \left(I\left(\mathbf{X}_{k} ; Y_{k}\right)\right. \\
& \left.+I\left(\mathbf{H}_{k}^{\top} \hat{\mathbf{X}}_{k} ;\left\{\mathbf{H}_{\ell}^{\top} \hat{\mathbf{X}}_{\ell}\right\}_{\ell=1}^{k-1} \mid \hat{\mathbf{X}}_{1}^{k}\right)\right)
\end{aligned}
$$

where the above mentioned lemma guarantees an input distribution with equal marginals and an average power of $\mathcal{E}$.

The first term is then upper-bounded by

$$
I\left(\mathbf{X}_{k} ; Y_{k}\right) \lesssim \mathrm{E}_{\hat{\mathbf{X}}_{k}}\left[\mathrm{C}_{\mathrm{SISO}, I I D, H=\mathbf{H}_{k}^{\top} \hat{\mathbf{X}}_{k}}(\mathcal{E})\right]
$$

where the approximation results from ignoring some additional terms that tend to zero as $\mathcal{E}$ tends to infinity. We hence get a 
bound

$$
\begin{aligned}
& \mathrm{C} \lesssim \mathrm{E}_{\hat{\mathbf{x}}_{-\infty}^{0}}\left[\mathrm{C}_{\mathrm{SISO}, \mathrm{IID}, H=\mathbf{H}_{0}^{\top} \hat{\mathbf{x}}_{0}}(\mathcal{E})\right. \\
&\left.\quad+I\left(\mathbf{H}_{0}^{\top} \hat{\mathbf{X}}_{0} ;\left\{\mathbf{H}_{\ell}^{\top} \hat{\mathbf{X}}_{\ell}\right\}_{\ell=-\infty}^{-1} \mid\left\{\hat{\mathbf{X}}_{\ell}=\hat{\mathbf{x}}_{\ell}\right\}_{\ell=-\infty}^{0}\right)\right] \\
& \leq \sup _{\hat{\mathbf{x}}_{-\infty}^{0}}\left\{\mathrm{C}_{\mathrm{SISO}, \mathrm{IID}, H=\mathbf{H}_{0}^{\top} \hat{\mathbf{x}}_{0}}(\mathcal{E})+I\left(\mathbf{H}_{0}^{\top} \hat{\mathbf{x}}_{0} ;\left\{\mathbf{H}_{\ell}^{\top} \hat{\mathbf{x}}_{\ell}\right\}_{\ell=-\infty}^{-1}\right\}\right.
\end{aligned}
$$

where the approximation results from ignoring the edge effects and the terms that will tend to zero as $\mathcal{E}$ tends to infinity.

The claim now follows by using the fading number of a memoryless SISO fading channel.

Next we state a lower bound to the fading number of a MISO fading channel:

Theorem 2: Consider a MISO fading channel with memory (5) where the stationary and ergodic fading process $\left\{\mathbf{H}_{k}\right\}$ takes value in $\mathbb{C}^{n_{\mathrm{T}}}$ and satisfies $h\left(\left\{\mathbf{H}_{k}\right\}\right)>-\infty$ and $\mathbf{E}\left[\left\|\mathbf{H}_{k}\right\|^{2}\right]<\infty$. Then the fading number $\chi\left(\left\{\mathbf{H}_{k}^{\top}\right\}\right)$ is lowerbounded by

$$
\begin{aligned}
\chi\left(\left\{\mathbf{H}_{k}^{\top}\right\}\right) \geq \sup _{\hat{\mathbf{x}}}\left\{\log \pi+\mathrm{E}\left[\log \left|\mathbf{H}_{0}^{\top} \hat{\mathbf{x}}\right|^{2}\right]\right. \\
\left.-h\left(\mathbf{H}_{0}^{\top} \hat{\mathbf{x}} \mid\left\{\mathbf{H}_{\ell}^{\top} \hat{\mathbf{x}}\right\}_{\ell=-\infty}^{-1}\right)\right\}
\end{aligned}
$$

where $\hat{\mathbf{x}} \triangleq \frac{\mathbf{x}}{\|\mathbf{x}\|}$ denotes a vector of unit length.

Moreover, this lower bound is achievable by IID inputs that can be expressed as the product of a constant unit vector $\hat{\mathbf{x}} \in$ $\mathbb{C}^{n_{\mathrm{T}}}$ and a circularly symmetric, scalar, complex IID random process $\left\{X_{k}\right\}$ such that

$$
\log \left|X_{k}\right|^{2} \sim \mathcal{U}([\log \log \mathcal{E}, \log \mathcal{E}]) .
$$

Note that this input satisfies the peak-power constraint (10) (and therefore also the average-power constraint (11).

Proof: We only give an outline of the proof. The details are omitted.

The lower bound is based on the assumption of a specific input distribution which is chosen to be of the form

$$
\mathbf{X}_{k}=X_{k} \cdot \hat{\mathbf{x}}
$$

where $\hat{\mathbf{x}}$ is a deterministic unit vector (the beam-direction) and where $\left\{X_{k}\right\}$ is IID circularly symmetric with

$$
\log \left|X_{k}\right|^{2} \sim \mathcal{U}([\log \log \mathcal{E}, \log \mathcal{E}]) .
$$

Note that this choice for $\left\{X_{k}\right\}$ achieves the fading number for the SISO fading channel

$$
Y_{k}=\left(\mathbf{H}_{k}^{\top} \hat{\mathbf{x}}\right) \cdot X_{k}+Z_{k}
$$

with fading process $\left\{H_{k}\right\}=\left\{\mathbf{H}_{k}^{\top} \hat{\mathbf{x}}\right\}$. The lower bound is then derived by proving

$$
\frac{1}{n} I\left(\mathbf{X}_{1}^{n} ; Y_{1}^{n}\right) \approx \frac{1}{n} \sum_{k=1}^{n} I\left(X_{k} ; Y_{k} \mid\left\{\mathbf{H}_{\ell}^{\top} \hat{\mathbf{x}}\right\}_{\ell=1}^{k-1}\right)
$$

and using the results of memoryless SISO fading channels with side-information [1], [2].

\section{Special Case of Isotropically Distributed FADING}

We next consider the special case of isotropically distributed fading processes, i.e., for every deterministic unitary $n_{\mathrm{T}} \times n_{\mathrm{T}}$ matrix $U$

$$
\mathbf{H}_{k} \stackrel{\mathscr{L}}{=} \mathbf{U H}_{k},
$$

where we use " $=\underline{=}$ " to denote equal in law.

In this case we have the following corollary:

Corollary 3: Consider a MISO fading channel with memory (5) where the stationary and ergodic fading process $\left\{\mathbf{H}_{k}\right\}$ takes value in $\mathbb{C}^{n_{\mathrm{T}}}$, satisfies $h\left(\left\{\mathbf{H}_{k}\right\}\right)>-\infty$ and $\mathrm{E}\left[\left\|\mathbf{H}_{k}\right\|^{2}\right]<\infty$, and is isotropically distributed. Then the upper bound (14) and the lower bound (19) coincide and the fading number $\chi_{\text {iso }}\left(\left\{\mathbf{H}_{k}^{\top}\right\}\right)$ is given by

$$
\begin{aligned}
\chi_{\text {iso }}\left(\left\{\mathbf{H}_{k}^{\top}\right\}\right)= & \log \pi+\mathbf{E}\left[\log \left|\mathbf{H}_{0}^{\top} \hat{\mathbf{e}}\right|^{2}\right] \\
& -h\left(\mathbf{H}_{0}^{\top} \hat{\mathbf{e}} \mid\left\{\mathbf{H}_{\ell}^{\top} \hat{\mathbf{e}}\right\}_{\ell=-\infty}^{-1}\right)
\end{aligned}
$$

where $\hat{\mathbf{e}}$ is any deterministic unit vector.

Proof: This follows immediately from Theorem 1 and 2 by noting that for any $\hat{\mathbf{e}}$

$$
\mathbf{H}_{k}^{\top} \hat{\mathbf{e}} \stackrel{\mathscr{L}}{=} \mathbf{H}_{k}^{\top} \mathbf{U}^{\top} \hat{\mathbf{e}}=\mathbf{H}_{k}^{\top} \hat{\mathbf{e}}^{\prime}
$$

where the first equality in law follows from 25 and the second equality by defining a new unit vector $\hat{\mathbf{e}}^{\prime} \triangleq \mathrm{U}^{\top} \hat{\mathbf{e}}$. Note that for the MISO case isotropically distributed is equivalent to rotation commutative in the generalized sense as defined in [1, Defintion 4.37] or [2, Defintion 6.37].

\section{Special Case of Gaussian Fading}

In this section we assume that the fading process $\left\{\mathbf{H}_{k}\right\}$ is a mean-d Gaussian process such that $\left\{\tilde{\mathbf{H}}_{k}\right\}=\left\{\mathbf{H}_{k}-\mathbf{d}\right\}$ is a zero-mean, circularly symmetric, stationary, ergodic, complex Gaussian process with matrix-valued spectral distribution function $F(\cdot)$, and with covariance matrix K. Furthermore, we assume that the prediction error covariance matrix $\Sigma$ is nonsingular (regularity assumption).

\section{A. Upper Bound for Gaussian Fading}

We start with a new derivation of the upper bound (3) based on Theorem 1 We will see that (3) is in general less tight than (14).

We start by loosening the upper bound (14) as follows:

$$
\begin{gathered}
\chi\left(\left\{\mathbf{H}_{k}^{\top}\right\}\right) \\
\leq \sup _{\hat{\mathbf{x}}_{-\infty}^{0}}\left\{\log \pi+\mathrm{E}\left[\log \left|\mathbf{H}_{0}^{\top} \hat{\mathbf{x}}_{0}\right|^{2}\right]\right. \\
\left.\quad-h\left(\mathbf{H}_{0}^{\top} \hat{\mathbf{x}}_{0} \mid\left\{\mathbf{H}_{\ell}^{\top} \hat{\mathbf{x}}_{\ell}\right\}_{\ell=-\infty}^{-1}\right)\right\} \\
=\sup _{\hat{\mathbf{x}}_{-\infty}^{0}}\left\{\log \pi+\mathrm{E}\left[\log \left|\mathbf{H}_{0}^{\top} \hat{\mathbf{x}}_{0}\right|^{2}\right]-h\left(\mathbf{H}_{0}^{\top} \hat{\mathbf{x}}_{0}\right)\right. \\
\left.\quad+h\left(\mathbf{H}_{0}^{\top} \hat{\mathbf{x}}_{0}\right)-h\left(\mathbf{H}_{0}^{\top} \hat{\mathbf{x}}_{0} \mid\left\{\mathbf{H}_{\ell}^{\top} \hat{\mathbf{x}}_{\ell}\right\}_{\ell=-\infty}^{-1}\right)\right\} \\
\leq \sup _{\hat{\mathbf{x}}_{0}}\left\{\log \pi+\mathrm{E}\left[\log \left|\mathbf{H}_{0}^{\top} \hat{\mathbf{x}}_{0}\right|^{2}\right]-h\left(\mathbf{H}_{0}^{\top} \hat{\mathbf{x}}_{0}\right)\right\}
\end{gathered}
$$




$$
\begin{aligned}
& +\sup _{\hat{\mathbf{x}}_{-\infty}^{0}}\left\{h\left(\mathbf{H}_{0}^{\top} \hat{\mathbf{x}}_{0}\right)-h\left(\mathbf{H}_{0}^{\top} \hat{\mathbf{x}}_{0} \mid\left\{\mathbf{H}_{\ell}^{\top} \hat{\mathbf{x}}_{\ell}\right\}_{\ell=-\infty}^{-1}\right)\right\} \\
= & \sup _{\hat{\mathbf{x}}_{0}}\left\{\log \pi+\mathrm{E}\left[\log \left|\mathbf{H}_{0}^{\top} \hat{\mathbf{x}}_{0}\right|^{2}\right]-h\left(\mathbf{H}_{0}^{\top} \hat{\mathbf{x}}_{0}\right)\right\} \\
& +\sup _{\hat{\mathbf{x}}_{-\infty}^{0}} I\left(\mathbf{H}_{0}^{\top} \hat{\mathbf{x}}_{0} ;\left\{\mathbf{H}_{\ell}^{\top} \hat{\mathbf{x}}_{\ell}\right\}_{\ell=-\infty}^{-1}\right),
\end{aligned}
$$

where (30) follows from

$$
\sup _{x}\{f(x)+g(x)\} \leq \sup _{x} f(x)+\sup _{x} g(x) .
$$

In [1, Corollary 4.28], [2, Corollary 6.28] it has been shown that the IID MISO fading number (1) for Gaussian fading is given by

$$
\begin{aligned}
\chi\left(\mathbf{H}^{\top}\right) & =\sup _{\|\hat{\mathbf{x}}\|=1}\left\{\log \pi+\mathrm{E}\left[\log \left|\mathbf{H}^{\top} \hat{\mathbf{x}}\right|^{2}\right]-h\left(\mathbf{H}^{\top} \hat{\mathbf{x}}\right)\right\} \\
& =-1+\log d_{*}^{2}-\operatorname{Ei}\left(-d_{*}^{2}\right)
\end{aligned}
$$

where $d_{*}$ is given in (4). This proves the equivalence of the first supremum in (31) with the first three terms of (3). It therefore only remains to prove that

$$
\sup _{\hat{\mathbf{x}}_{-\infty}^{0}} I\left(\mathbf{H}_{0}^{\top} \hat{\mathbf{x}}_{0} ;\left\{\mathbf{H}_{\ell}^{\top} \hat{\mathbf{x}}_{\ell}\right\}_{\ell=-\infty}^{-1}\right) \leq \log \frac{\|\mathrm{K}\|}{\lambda_{\min }} .
$$

To this goal note that

$$
\begin{aligned}
\sup _{\hat{\mathbf{x}}_{-\infty}^{0}} I\left(\mathbf{H}_{0}^{\top} \hat{\mathbf{x}}_{0} ;\left\{\mathbf{H}_{\ell}^{\top} \hat{\mathbf{x}}_{\ell}\right\}_{\ell=-\infty}^{-1}\right) \\
\quad \leq \sup _{\hat{\mathbf{x}}_{-\infty}^{0}} I\left(\mathbf{H}_{0}^{\top} \hat{\mathbf{x}}_{0} ;\left\{\mathbf{H}_{\ell}^{\top} \hat{\mathbf{x}}_{\ell}\right\}_{\ell=-\infty}^{-1}, \mathbf{H}_{-\infty}^{-1}\right) \\
=\sup _{\hat{\mathbf{x}}_{0}} I\left(\mathbf{H}_{0}^{\top} \hat{\mathbf{x}}_{0} ; \mathbf{H}_{-\infty}^{-1}\right) \\
=\sup _{\hat{\mathbf{x}}_{0}}\left\{h\left(\mathbf{H}_{0}^{\top} \hat{\mathbf{x}}_{0}\right)-h\left(\mathbf{H}_{0}^{\top} \hat{\mathbf{x}}_{0} \mid \mathbf{H}_{-\infty}^{-1}\right)\right\} \\
=\sup _{\hat{\mathbf{x}}_{0}}\left\{\log \left(\pi e \hat{\mathbf{x}}_{0}^{\dagger} \mathbf{K} \hat{\mathbf{x}}_{0}\right)-h\left(\mathbf{H}_{0}^{\top} \hat{\mathbf{x}}_{0} \mid \mathbf{H}_{-\infty}^{-1}\right)\right\} .
\end{aligned}
$$

Here, the first inequality follows from the inclusion of additional random variables in the mutual information; the subsequent equality from the fact that given the past realization of the fading, $\mathbf{H}_{0}^{\top} \hat{\mathbf{x}}_{0}$ is independent of $\left\{\mathbf{H}_{\ell}^{\top} \hat{\mathbf{x}}_{\ell}\right\}_{\ell=-\infty}^{-1}$; and in the last equality we have used the expression for the differential entropy of a Gaussian random variable with $\mathrm{K}$ denoting the covariance matrix of $\left\{\mathbf{H}_{k}\right\}$.

Note that the first inequality in general is not tight, i.e., (3) is in general looser than 31 which in turn is in general looser than (14).

To compute the second term on the RHS of (39), we express the fading $\mathbf{H}_{0}$ as

$$
\mathbf{H}_{0}=\overline{\mathbf{H}}_{0}+\tilde{\mathbf{H}}_{0}
$$

with $\overline{\mathbf{H}}_{0}$ being the best estimate of $\mathbf{H}_{0}$ based on the past realizations. We note that $\tilde{\mathbf{H}}_{0} \sim \mathcal{N}_{\mathbb{C}}(\mathbf{0}, \Sigma)$ where $\Sigma$ denotes the prediction error covariance matrix. Hence

$$
h\left(\mathbf{H}_{0}^{\top} \hat{\mathbf{x}}_{0} \mid \mathbf{H}_{-\infty}^{-1}\right)=\log \left(\pi e \hat{\mathbf{x}}_{0}^{\dagger} \Sigma \hat{\mathbf{x}}_{0}\right) .
$$

The bound 35 now follows by the Rayleigh-Ritz Theorem [7, Theorem 4.2.2], [2, Theorem A.9]

$$
\lambda_{\min }=\min _{\hat{\mathbf{x}}} \hat{\mathbf{x}}^{\dagger} \Sigma \hat{\mathbf{x}},
$$

the definition of the Euclidean norm of matrices, and the properties of positive semi-definite matrices:

$$
\max _{\hat{\mathbf{x}}} \hat{\mathbf{x}}^{\dagger} \mathrm{K} \hat{\mathbf{x}}=\max _{\hat{\mathbf{x}}} \hat{\mathbf{x}}^{\dagger} S^{\top} S \hat{\mathbf{x}}=\max _{\hat{\mathbf{x}}}\|S \hat{\mathbf{x}}\|^{2}=\|S\|^{2}=\|\mathrm{K}\| .
$$

\section{B. Spatially IID Gaussian Fading}

We next specialize the assumptions to the case where $\left\{\tilde{\mathbf{H}}_{k}\right\}=\left\{\mathbf{H}_{k}-\mathbf{d}\right\}$ is a spatially IID process where each component is a zero-mean unit-variance circularly symmetric complex Gaussian process of spectral distribution function $\mathrm{F}(\cdot)$. For this case we will now present a new derivation of the result (2) based on our new bounds.

Note that we cannot apply Corollary 3 here: even though $\left\{\tilde{\mathbf{H}}_{k}\right\}$ is isotropically distributed, $\left\{\mathbf{H}_{k}\right\}$ is not due to its mean vector $\mathbf{d}$.

However, the term $I\left(\mathbf{H}_{0}^{\top} \hat{\mathbf{x}}_{0} ;\left\{\mathbf{H}_{\ell}^{\top} \hat{\mathbf{x}}_{\ell}\right\}_{\ell=-\infty}^{-1}\right)$ does not depend on the particular choice of $\hat{\mathbf{x}}_{\ell}$ :

$$
\begin{aligned}
I & \left(\mathbf{H}_{0}^{\top} \hat{\mathbf{x}}_{0} ;\left\{\mathbf{H}_{\ell}^{\top} \hat{\mathbf{x}}_{\ell}\right\}_{\ell=-\infty}^{-1}\right) \\
& =I\left(\mathbf{H}_{0}^{\top} \hat{\mathbf{x}}_{0}-\mathbf{d}^{\top} \hat{\mathbf{x}}_{0} ;\left\{\mathbf{H}_{\ell}^{\top} \hat{\mathbf{x}}_{\ell}-\mathbf{d}^{\top} \hat{\mathbf{x}}_{\ell}\right\}_{\ell=-\infty}^{-1}\right) \\
& =I\left(\tilde{\mathbf{H}}_{0}^{\top} \hat{\mathbf{x}}_{0} ;\left\{\tilde{\mathbf{H}}_{\ell}^{\top} \hat{\mathbf{x}}_{\ell}\right\}_{\ell=-\infty}^{-1}\right) \\
& =I\left(\tilde{\mathbf{H}}_{0}^{\top} \hat{\mathbf{e}} ;\left\{\tilde{\mathbf{H}}_{\ell}^{\top} \hat{\mathbf{e}}\right\}_{\ell=-\infty}^{-1}\right) \\
& =I\left(H_{0}^{(1)} ;\left\{H_{\ell}^{(1)}\right\}_{\ell=-\infty}^{-1}\right) \\
& =\log \frac{1}{\epsilon^{2}} .
\end{aligned}
$$

Equation (2) now follows from [34, Theorem 11 and Theorem 2 by noting that

$$
\max _{\|\hat{\mathbf{x}}\|=1} \frac{\left|\mathrm{E}\left[\mathbf{H}_{k}^{\top}\right] \hat{\mathbf{x}}\right|}{\sqrt{\operatorname{Var}\left(\mathbf{H}_{k}^{\top} \hat{\mathbf{x}}\right)}}=\max _{\|\hat{\mathbf{x}}\|=1}\left|\mathbf{d}^{\top} \hat{\mathbf{x}}\right|=\|\mathbf{d}\|,
$$

where the maximum is achieved for $\hat{\mathbf{x}}=\mathbf{d} /\|\mathbf{d}\|$.

\section{DISCUSSION \& CONCLUSION}

We have derived two bounds for a MISO fading channel of general law including memory. Both bounds show the same structure involving the maximization of a deterministic beamdirection $\hat{\mathbf{x}}$, which suggests that beam-forming is optimal at high SNR. However, one has to be aware that the beamdirection is not chosen to maximize the SNR, but to maximize the fading number.

The differences between the upper and lower bound lies in the details of the maximization: while in the lower bound one single direction unit vector $\hat{\mathrm{x}}$ is chosen for all time, the upper bound allows for different realizations of $\hat{\mathbf{x}}_{k}$ for different times $k$.

We are convinced that the lower bound is actually tight: intuition tells that for our stationary channel model a stationary input should be sufficient for achieving the capacity. As a matter of fact in the SISO and SIMO case it has been shown that actually an IID input suffices to achieve capacity at high 
SNR [1], [2], [4]. Furthermore, we have been able to modify the derivation of the upper bound such as to get the following bound:

$$
\begin{aligned}
& \chi\left(\left\{\mathbf{H}_{k}^{\top}\right\}\right) \\
& \leq \sup _{Q_{\hat{\mathbf{x}}_{-\kappa}, \ldots, \hat{\mathbf{x}}_{0}} \in \mathcal{P}^{*}} \mathrm{E}_{\hat{\mathbf{X}}_{-\kappa}^{0}}\left[\log \pi+\mathrm{E}\left[\mathbf{H}_{0}^{\top} \hat{\mathbf{X}}_{0} \mid \hat{\mathbf{X}}_{0}\right]\right. \\
& \left.\quad-h\left(\mathbf{H}_{0}^{\top} \hat{\mathbf{X}}_{0} \mid\left\{\mathbf{H}_{\ell}^{\top} \hat{\mathbf{X}}_{\ell}\right\}_{\ell=-\kappa}^{-1},\left\{\hat{\mathbf{X}}_{\ell}=\hat{\mathbf{x}}_{\ell}\right\}_{\ell=-\kappa}^{0}\right)\right] .
\end{aligned}
$$

Here, $\kappa$ is an arbitrary (large) positive integer, and $\mathcal{P}^{*}$ denotes the set of all joint distributions on $\hat{\mathbf{X}}_{-\kappa}, \ldots, \hat{\mathbf{X}}_{0}$ where

$$
\left\|\hat{\mathbf{X}}_{\ell}\right\|=1 \quad \text { a.s. }
$$

and where each $\hat{\mathbf{X}}_{\ell}$ has the same distribution, for all $\ell=$ $-\kappa, \ldots, 0$. This bound can easily be loosened to get the result of Theorem 11 one simply upper-bounds the supremum over $Q$ with a supremum over $\hat{\mathbf{x}}_{-\kappa}^{0}$. However, it intuitively seems that under the constraint that all marginals of $Q$ must be the same this bound is not tight.

In the case of isotropically distributed fading the particular choice of direction has no influence on the fading process and therefore the upper and lower bounds coincide.

In the case of Gaussian fading we could show that the bounds presented in [5] and [6] are special cases of the new bounds presented here, where the new upper bound (14) is in general tighter than (3).

The success of further attempts on deriving the MISO fading number precisely will be crucial to the investigation of the fading number of general MIMO fading channels.

\section{ACKNOWLEDGMENTS}

Helpful comments from Amos Lapidoth, Tobias Koch, and Daniel Hösli are gratefully acknowledged.

\section{REFERENCES}

[1] A. Lapidoth and S. M. Moser, "Capacity bounds via duality with applications to multiple-antenna systems on flat fading channels," IEEE Transactions on Information Theory, vol. 49, no. 10, pp. 2426-2467, October 2003.

[2] S. M. Moser, "Duality-based bounds on channel capacity," Ph.D. dissertation, Swiss Federal Institute of Technology, Zurich, October 2004, Diss. ETH No. 15769. [Online]. Available: http://www.isi.ee.ethz.ch/\$“sim\$moser

[3] A. Lapidoth and S. M. Moser, "The fading number of SIMO fading channels with memory," in Proceedings IEEE International Symposium on Information Theory and its Applications (ISITA), Parma, Italy, October 10-13, 2004, pp. 287-292.

[4] _ , "The fading number of single-input multiple-output fading channels with memory," November 2004, to appear in IEEE Transactions on Information Theory.

[5] T. Koch and A. Lapidoth, "Degrees of freedom in non-coherent stationary MIMO fading channels," in Proceedings Winter School on Coding and Information Theory, Bratislava, Slovakia, February 20-25, 2005, pp. 9197.

[6] - "The fading number and degrees of freedom in non-coherent MIMO fading channels: a peace pipe," in Proceedings IEEE International Symposium on Information Theory (ISIT), Adelaide, Australia, September 4-9, 2005, pp. 661-665.

[7] R. A. Horn and C. R. Johnson, Matrix Analysis. Cambridge University Press, 1985. 